\title{
EXPONENTIAL SUMS AND FORMS FOR VARIETIES OVER FINITE FIELDS
}

\author{
RICHARD G. SARKISIAN
}

\begin{abstract}
We prove that the roots of the $L$-function of an Artin-Schreier cover of an algebraic variety defined over a finite field differ from the roots of the zeta function of the cover by roots of unity.
\end{abstract}

In this paper we use an observation about forms for varieties to prove that the roots of the $L$-function of an Artin-Schreier cover of an algebraic variety defined over a finite field differ from the roots of the zeta function of the cover by roots of unity. This answers affirmatively a conjecture of Bombieri [1].

More specifically, let $X$ be an absolutely irreducible normal variety defined over the finite field $k=\mathbf{F}_{q}$ with $q=p^{a}$ elements. Let $\bar{k}$ be an algebraic closure of $k$, and $k(X), \bar{k}(X)$ the function field of $X$ over $k$ and $\bar{k}$, respectively. Let $R(x) \in k(X)$ be such that $R(x) \neq h^{p}-h$ for $h \in \bar{k}(X)$. Let $X_{m}$ be the set of points of $X$ defined over $\mathbf{F}_{q^{m}}$, and let

$$
S_{m}(R, X)=\sum^{\prime} \exp [2 \pi i t r(R(x)) / p]
$$

where the sum is over $x \in X_{m}$, the 'means exclude the points lying on the poles of $R(x)$, and $\operatorname{tr}: \mathbf{F}_{q} \rightarrow \mathbf{F}_{p}$ is the absolute trace.

Let $Y$ be the variety defined by $Z^{p}-Z=R(x)$. Then there exists a Galois covering $\pi: Y \rightarrow X$ sending $(z, x) \rightarrow x$. This covering has Galois group $\mathbf{F}_{p}$ acting on $Y$ by $(z, x) \rightarrow(z+g, x), g \in \mathbf{F}_{p} . \pi$ is called an Artin-Schreier covering of $X$ related to $R(x)$. Define the $L$-function of this covering by

$$
L(t, R, X)=\exp \left(\sum_{m=1}^{\infty} S_{m}(R, X) t^{m} / m\right) .
$$

For further information see the paper of Bombieri [1].

Let $Z(t, Y, q)$ denote the zeta function of $Y$ relative to the field of definition $k$. Bombieri [1, p. 105] conjectured that if $\theta$ is a characteristic root, i.e. a zero or pole of $L(t, R, X)$, then there exists a characteristic root $\omega$ of $Z(t, Y, q)$ and a $p^{u}$ th root of unity $\gamma$ for some integer $u$ such that $\theta=\gamma \omega$. In this paper we prove this conjecture.

We first recall a theorem of Bombieri [1, Theorem 7]. Let $Y(\lambda)$ be the ArtinSchreier cover associated to $Z^{p}-Z=R(x)+\lambda$ where $\lambda \in \mathbf{F}_{q^{p}}$.

Received by the editors November 19, 1984.

1980 Mathematics Subject Classification. Primary 10G05.

Key words and phrases. Exponential sum, Artin-Schreier cover, form for an algebraic variety.

(C)1985 American Mathematical Society $0002-9939 / 85 \$ 1.00+\$ .25$ per page 
THEOREM 1 (BOMBIERI [1]). $L(t, R, X)$ is a rational function of $t$. There exists a suitable power $p^{u}$ of the degree $p$ of the cover $\pi: Y \rightarrow X$ such that, if $\theta$ is $a$ characteristic root of $L(t, R, X)$, then there exists $v \leqslant u-1$ and $\lambda \in \mathbf{F}_{q^{p}}$ such that $\theta^{p^{u}}=w^{p^{u-1}}$ where $w$ is a characteristic root of $Z\left(t, Y(\lambda), q^{p^{\prime \prime}}\right)$.

Let $k^{\prime}$ be the extension of $k$ of degree $s$.

DEFINITION 1. Let $V$ be an algebraic variety over $k$. A $k^{\prime} / k$-form for $V$ is a pair $\left(V^{\prime}, f\right)$ where $V^{\prime}$ is an algebraic variety over $k$ and $f: V^{\prime} \rightarrow V$ is an isomorphism defined over $k^{\prime}$.

It is easy to see that, if $\left(V^{\prime}, f\right)$ is a $k^{\prime} / k$-form for $V$, then the characteristic roots of $Z\left(t, V^{\prime}, q\right)$ differ from those of $Z(t, V, q)$ by $s$ th roots of unity.

LEMMA 1. If $w$ is a characteristic root of $Z\left(t, Y(\lambda), q^{p^{n}}\right)$ and $\lambda \in \mathbf{F}_{q^{p}}$ is of the form $c^{p}-c$ for some $c \in \mathbf{F}_{q^{p^{\prime+1}}}$, then $w^{p}$ is a characteristic root of $Z\left(t, Y, q^{p^{n+1}}\right)$.

Proof. $Z^{p}-Z=R(x)+\lambda=R(x)+c^{p}-c$, i.e. $(Z-c)^{p}-(Z-c)=R(x)$. Thus, $Y(\lambda)$ and $Y$ are $\mathbf{F}_{q^{p^{k}+1}} / \mathbf{F}_{q^{p^{p}}}$-forms. Therefore, $\zeta w$ is a characteristic root of $Z\left(t, Y, q^{p^{\prime}}\right)$ where $\zeta$ is some $p$ th root of unity. Therefore $w^{p}$ is a characteristic root of $Z\left(t, Y, q^{p^{n+1}}\right)$. This proves the lemma.

Now observe that $\left(w^{p}\right)^{p^{-r-1}}=w^{p^{-r}}$ is a characteristic root of $Z\left(t^{p^{t+1}}, Y, q^{p^{k+1}}\right)$. But

$$
Z\left(t^{p^{\prime+1}}, Y, q^{p^{\prime+1}}\right)=\prod_{\zeta} Z(\zeta t, Y, q)
$$

where the product is over all $p^{v+1}$ th roots of unity. Therefore we must have that $w^{p^{-n}}$ is a characteristic root of one of the factors $Z(\zeta t, Y, q)$. This proves the following lemma.

LEMMA 2. If $w$ is a characteristic root of $Z\left(t, Y(\lambda), q^{p^{n}}\right)$, then $w^{p^{-n}}=\zeta r$ where $r$ is a characteristic root of $Z(t, Y, q)$ and $\zeta$ is a $p^{v+1}$ th root of unity.

Now, by Theorem 1 , we have that if $\theta$ is a characteristic root of $L(t, R, X)$, then

$$
\theta^{p^{\prime \prime}}=w^{p^{u-v}}=\left(w^{p^{-r}}\right)^{p^{u}}, \quad v \leqslant u-1,
$$

where $w$ is a characteristic root of $Z\left(t, Y(\lambda), q^{p^{\prime \prime}}\right)$ for some $\lambda \in \mathbf{F}_{q^{p^{\prime \prime}}}$ of the form $c^{p}-c, c \in \mathbf{F}_{q^{p^{p+1}}}$. Taking $p^{u}$ th roots we get

$$
\theta=\eta w^{p^{-r}}=(\eta \zeta) r
$$

where $\eta$ is a $p^{u}$ th root of unity, $\zeta$ is a $p^{v+1}$ th root of unity and $r$ is a characteristic root of $Z(t, Y, q)$. Since $v+1 \leqslant u, \eta \zeta$ is a $p^{u}$ th root of unity. Letting $\gamma=\eta \zeta, \omega=r$ we have the following theorem.

THEOREM 2. If $\theta$ is a characteristic root of the $L$-function $L(t, R, X)$ associated to the Artin-Schreier cover $Y: Z^{p}-Z=R(x)$ of $X$, then $\theta=\gamma \omega$ where $\gamma$ is a $p^{u}$ th root of unity and $\omega$ is a characteristic root of $Z(t, Y, q)$. 
ACKNOWLEDGEMENT. This paper is a part of the author's 1984 CUNY Graduate Center Doctoral Dissertation written under the direction of Raymond T. Hoobler. I would also like to thank Noriko Yui for reading the manuscript and making useful suggestions.

\section{REFERENCES}

1. E. Bombieri, On exponential sums in finite fields, Amer. J. Math. 88 (1966), 71-105.

Department of Mathematics, University of Toronto, Toronto, Ontario, Canada M5S 1A1 